\title{
A second Human Genome Project?
}

With only $4 \%$ of the Human Genome Project completed, albeit ahead of schedule, one might conclude that the so-called genomics revolution has quite a bit more time on the meter. But there is another aspect of the human genome that we can no longer afford to ignore: The more than three million differences in the genetic code that uniquely identify each one of us. Not much when one considers a genome of over three billion bases, but of sufficent clinical importance to forever change the face of human healthcare and propel forward the field of pharmacogenomics.

Who should know better than Francis Collins, director of the US National Human Genome Research Institute, who recently helped Novartis initiate a summit meeting with their pharmaceutical brethren to discuss the joint funding of a single nucleotide polymorphism (SNP) mapping effort? According to Collins, a publicly available SNP map will be critical to understanding how to use the data that is generated by the Human Genome Project. A catalog of all common human sequence variations will enable the identification of weaker polygenic contributors to disease, the design of personalized prognostic strategies, and ultimately the adoption of individually optimized therapies- the Holy Grail of pharmacogenomic research.

In May, TIGR's Craig Venter declared his intent to mount a massive effort to sequence and collect SNPs, forming with Perkin-Elmer the $\$ 200$ million joint venture Celera Genomics. Venter has an acute awareness of the cutting edge in allying technology to science: If he is not the first to start something, he will do it better or faster. (In the case of Celera, he claims he can resequence the human genome ten times over and seven years ahead of the public effort). The fact that Venter has put a high priority on mapping SNPs is significant.

And now Incyte has launched an initiative. For a mere "snip" (\$38 million), it has acquired Hexagen - a UK company with a proprietary high-throughput SNP scanning system-and intends to invest over the next two years a staggering $\$ 200$ million in an effort to sequence the protein-coding regions of the human genome and "gather SNP data for every human gene."

So it seems that Daniel Cohen and Genset got the concept right a year ago when they announced their effort to create a biallelic map of the entire human genome. Now the race is on to identify SNPs of clinical relevance-perhaps a trickier proposition than many of the companies involved would like us to believe, particularly as the technologies are unproven and the existing data preliminary. But with the US National Institute of General Medical Sciences promising to galvanize government-sponsored research in the area ${ }^{*}$ and an influential nucleus of private companies investing significant resources, there are interesting parallels with the early days of genomics. Back then, it took private and public money to kickstart the field. In the light of recent events, the move into SNP mapping and pharmacogenomics - the second Human Genome Project if you will-should be as swift and dramatic as the events that followed the "original" Human Genome Project.

*Rochelle Long, chief of the Pharmacological \& Physiological Sciences Branch, wil present NIGMS' plans at Nature Biotechnology's Validating Pharmacogenomics meeting next month (October 1-2, 1998).

\section{Beat bioterror with batch science}

The US government's multimillion dollar antibioterrorism plan to stockpile vaccines and/or antibiotics at strategic locations around the country in order to protect civilian populations in the event of a bioweapons attack won't work. It is the equivalent of having the citizenry rush to their basements and throw their arms up over their heads during a nuclear war-another government plan that served only to distract us from the knowledge that we would have little control over our fates should such an event occur.

The Clinton vaccine plan-drawn up in haste and in reaction to renewed clashes with Iraq's Sadam Hussein over UN inspections of his purported bioweapons facilities-has a dubious future now that the dust of imminent threat has cleared. Although biotechnology could certainly take advantage of this short-sighted strategy, it has a responsibility-and a vested interest--to take the longer view and work toward the development of realistic plans that have at least a chance of being effective.

In this issue, Scott Layne and Tony Beugelsdijk present a proposal for "batch science," (see "Laboratory firepower for infectious disease research," p. 825) that could serve as the basis for more integrated solutions to dealing with multifaceted problems like bioterrorism. Batch science is an approach to solving problems that require large amounts of data analysis by linking laboratories over the Internet for problem solving both in real and nonreal time. Batch science machines can serve as programmable laboratory technicians, performing the mechanical work of hundreds of human beings.

One example of batch science discussed by the authors is the typing of influenza A viruses by an automated reference library. To carry out various procedures, flu investigators would use a suite of process control tools to program manage and track procedures at every step. With respect to bioweapons, batch science facilities could be used to monitor, inspect, and test for infectious agents in a timely manner, to offer timely information in the event of an attack-How many agents were released? How do they differ? What is the best treatment for those affected?-and to assist in the aftermath, by identifying and categorizing lethal agents and helping to determine who made them. The authors believe that the creation of batch science facilities would not require a biological "Manhattan project," and that first-generation facilities could be up and running in 2-3 years.

A system such as the one they describe is an attempt at creating a coordinated plan for dealing with big problems-like biological warfare-that require big biological solutions. The medical community has long expressed its interest and involvement in finding solutions to problems posed by biological and biotechnology-driven threats. It's time for the biotechnology industry--which will make the vaccines and the antibiotics and the diagnostics that will protect and defend us from these threats - to do the same. 